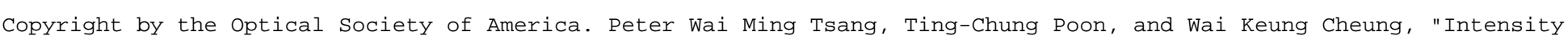

image-embedded binary holograms," Appl. Opt. 52, A26-A32 (2013); doi: 10.1364/AO.52.000A26

\title{
Intensity image-embedded binary holograms
}

\author{
Peter Wai Ming Tsang, ${ }^{1}$ Ting-Chung Poon, ${ }^{2,3}$ and Wai Keung Cheung ${ }^{1, *}$ \\ 'Department of Electronic Engineering, City University of Hong Kong, Hong Kong, China \\ ${ }^{2}$ Bradley Department of Electrical and Computer Engineering, MC 0111, 1185 Perry Street, \\ Room 302, Virginia Tech, Blacksburg, Virginia 24061, USA \\ ${ }^{3}$ Shanghai Institute of Optics and Fine Mechanics, Chinese Academy of Sciences, \\ P.O. Box 800-211, Shanghai 201800, China \\ *Corresponding author: $50695250 @$ student.cityu.edu.hk
}

Received 20 July 2012; revised 12 September 2012; accepted 12 September 2012; posted 13 September 2012 (Doc. ID 173012); published 11 October 2012

\begin{abstract}
Past research has demonstrated that, by downsampling the source object scene in multiple directions, a binary Fresnel hologram can be generated to preserve favorable quality on the reconstructed image. In this paper, we will show that a binary hologram generated with such an approach is also insensitive to noise contamination. On this basis, we propose a method to embed an intensity image into the binary hologram. To prevent the embedded information from being tampered or retrieved with unauthorized means, scrambling is applied to relocate each pixel to a unique position in the binary hologram according to a random assignment that is only known with the availability of a descrambling key. Experimental results demonstrate that our proposed method is capable of embedding an intensity image that is one quarter the size of the binary hologram without causing observable degradation on the reconstructed image. In addition, the embedded image can be retrieved with acceptable quality even if the binary hologram is damaged and contaminated with noise. () 2012 Optical Society of America

OCIS codes: $\quad 090.0090,090.1760,090.1995$.
\end{abstract}

\section{Introduction}

Investigation on the generation of a binary Fresnel hologram has been an area of interest in the realm of digital holography [1]. Comparing with the graylevel holograms, a binary hologram has two major advantages. First, the data size is reduced considerably as each hologram pixel is represented by a single bit. Second, a binary hologram can be hard-copied with high-resolution printers (such as laser printers and image setters) [2], which generally could only support printing of black or white dots. This provides an alternative and more economical means for printing a digital hologram without the need of the sophisticated fringe printer [3]. The pioneer work can be dated back to the $1960 \mathrm{~s}[4,5]$ when a successful attempt in the realization of binary Fraunhofer

1559-128X/13/010A26-07\$15.00/0

(C) 2013 Optical Society of America holograms was demonstrated. Later, methods on the generation of binary holograms in an iterative manner have been reported in [6-8]. Moderate success has also been attained in applying error diffusion [9] in the binarization of Fourier [10-12] and Fresnel [13] holograms. Despite the swiftness and effectiveness of the error diffusion method, the optical reconstructed images of holograms binarized with such means are weak and contaminated with noise. Recently, Tsang and coworkers [14,15] have proposed a fast, noniterative method for generating binary holograms, which results in reconstructed images with favorable visual qualities. In this approach, a threedimensional (3D) scene is first downsampled with a uniform cross [14] or grid-cross [15] lattice prior to the generation of the hologram. The latter is then binarized with sign thresholding, where each pixel is converted into black and white dots according to its polarity. As will be shown in the later part of this paper, a binary hologram generated with this method 
is highly resistant to noise contamination. Based on these essential findings, we propose a method to embed one or more hidden intensity image(s) into the binary hologram. We have incorporated a scrambling scheme so that the content is hidden according to a random location mapping sequence that can only be retrieved with the availability of a descrambling key. In addition, experimental results demonstrate that, with our method, the embedded image(s) can be recovered with reasonable quality even if the binary hologram is contaminated with noise and damaged in certain region.

Research in the incorporation of holographic techniques in image hiding, sometimes referred to as "holographic watermarking," has been an area of interest for some time. In one approach, for example, the works reported in [16-19], a computer-generated hologram is embedded into an optical image (such as a picture or graphic art). In another approach, an optical image is embedded into a hologram. Our method belongs to the latter category. Although techniques for hiding images in gray holograms have been developed in the past (such as [20-22]), embedding additional information requires modifying the original intensities of certain pixels to different values within a dynamic range. As such, these methods are not applicable to a binary hologram as the intensity of each pixel in the latter can only be either black or white. Our method has the distinct advantage that pictorial information can be embedded by simply complementing a subset of pixels in the binary hologram. To our understanding, this is the first time where an intensity image has been proposed to be embedded in a binary hologram.

Organization of the paper is given as follows. For clarity of explanation, a brief outline on the binary hologram method in [14] and [15] is described in Section 2. We shall demonstrate the noise resistance of the binary hologram generated with such means. Our proposed method to embed pictorial content into the binary image is presented in Section $\underline{3}$. Experimental results to evaluate the propose $\bar{d}$ method are given in Section 4 , followed by a conclusion in Section $\underline{5}$ summarizing the essential findings.

\section{Computer Generation and Binarization of Fresnel Holograms}

In this section, we first describe briefly the binary hologram generation method reported in $[14,15]$. Next, the effect of noise contamination on the binary hologram derived with this method is evaluated. To begin with, we consider a 3D surface as shown in Fig. 1 . Each point on the 3D surface is assumed to be selfilluminating with an intensity profile $I(u, v)$. The distance of a point at position $(u, v)$ to the hologram plane $(x, y)$ along the $z$ direction is denoted by $w_{u ; v}$. As explained and demonstrated in [14], if a hologram is generated from the 3D surface and binarized with sign thresholding, assigning white and black intensities to positive and negative hologram pixels, respectively, the shaded region in the reconstructed image

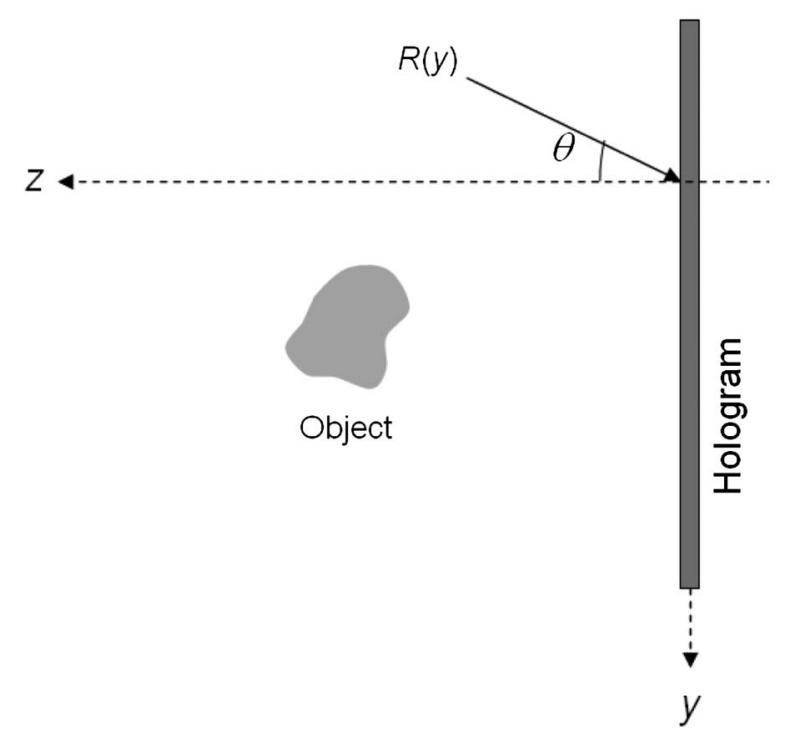

Fig. 1. Hologram illuminated with an inclined planar reference beam.

will be severely weakened. To overcome this problem, the intensity profile $I(u, v)$ is first subsampled into a new image $I_{D}(u, v)$ composed of four components as given by

$$
I_{D}(u, v)=I_{1}(u, v) \cup I_{2}(u, v) \cup I_{3}(u, v) \cup I_{4}(u, v) .
$$

The first pair of components, $I_{1}(u, v)$ and $I_{2}(u, v)$, is obtained through subsampling $I(u, v)$ with a grid lattice as

$$
\begin{aligned}
& I_{1}(u, v)=\left\{\begin{array}{cc}
I(u, v) & u=\tau M \\
0 & \text { otherwise }
\end{array}\right. \\
& I_{2}(u, v)=\left\{\begin{array}{cc}
I(u, v) & v=\tau M \\
0 & \text { otherwise }
\end{array}\right.
\end{aligned}
$$

The second pair of components, $I_{3}(u, v)$ and $I_{4}(u, v)$, is obtained through subsampling $I(u, v)$ with a cross lattice as

$$
\begin{gathered}
I_{3}(u, v)=\left\{\begin{array}{cc}
I(u, v) & (u \bmod M)=(v \bmod M) \\
0 & \text { otherwise }
\end{array}\right. \\
I_{4}(u, v)=\left\{\begin{array}{cc}
I(u, v) & (u \bmod M)=[(M-1)-(v \bmod M)] \\
0 & \text { otherwise }
\end{array}\right.
\end{gathered}
$$

where $\bmod$ is the modulo operator. The term $M$ is the subsampling factor that governs the sparseness of the subsampled image $I_{D}(u, v)$.

Next, the object wavefront $O(x, y)$ is derived by accumulating the contribution of individual object points in $I_{D}(u, v)$, each forming a Fresnel zone plate [1]: 


$$
\left.O(x, y)\right|_{0 \leq x<X}=\sum_{u=0}^{X-1} \sum_{v=0}^{Y-1} \frac{I_{D}(u, v) \exp \left(i 2 \pi r_{u ; v ; x ; y} / \lambda\right)}{r_{u ; v ; x ; y}}
$$

where $I_{D}(u, v)$ and $r_{u ; v ; x ; y}=\sqrt{(x-u)^{2}+(y-v)^{2}+w_{u ; v}^{2}}$ are the intensity of the point located at $(u, v)$ in the $3 \mathrm{D}$ scene and its distance to the hologram, respectively. $\lambda$ is the wavelength of the optical beam. $X$ and $Y$ are the horizontal and vertical extents of the hologram and, without loss of generality, assumed to be identical to that of the image scene. Subsequently, the object wavefront is mixed with a planar reference beam $R(y)$, and the real part is extracted to give an off-axis hologram $H(x, y)$, i.e.,

$$
H(x, y)=\operatorname{Re}[O(x, y) R(y)],
$$

where $\operatorname{Re}[\cdot]$ represents the real part of a complex number being bracketed. $R(y)$ is inclined at an angle of illumination $\theta$ along the vertical direction as shown in Fig. 1.

Subsequently, a binary hologram $H_{B}(x, y)$ is derived from $H(x, y)$ with sign thresholding as given by

$$
H_{B}(x, y)=\left\{\begin{array}{lc}
1 & \text { if } H(x, y) \geq 0 \\
0 & \text { otherwise }
\end{array} .\right.
$$

A binary hologram generated with this method results in favorable visual quality on the reconstructed image. As an example, a binary hologram of a single depth, $512 \times 512$ image shown in Fig. 2 a is generated based on Eqs. (7) and (8), having the optical setting in Table 1 .

The image is positioned at distances of $0.5 \mathrm{~m}$ from the hologram, and the numerical reconstructed images of the binary hologram is shown in Fig. $2 \mathrm{~b}$. It can be seen that the visual quality of the reconstructed image is favorable.

To illustrate the effect of noise resistance on the binary hologram, about $20 \%$ of the binary pixels are randomly selected, and each of them is complemented, i.e., $1 \mathrm{~s}$ become $0 \mathrm{~s}$ and $0 \mathrm{~s}$ become $1 \mathrm{~s}$. The reconstructed image of the noise-contaminated binary hologram is shown in Fig. 2c. Comparing the results in Figs. 2b and 2c, it can be seen that the degradation caused by the addition of noise is not prominent visually. The similarity between the two images, in terms of peak signal-to-noise ratio (PSNR), is $31.05 \mathrm{~dB}$, which is rather high and in line with the result based on visual inspection. Typically, a PSNR of around $28 \mathrm{~dB}$ in images is considered acceptable visually. To demonstrate this, the percentage of contaminated pixels (i.e., pixels that have been complemented) is increased to $30 \%$ to the binary hologram, and the reconstructed image is shown in Fig. 2d. We observe that the visual quality of the reconstructed image, with a PSNR of $27.9 \mathrm{~dB}$, is still acceptable, but the degradation caused by the higher percentage of noise contamination becomes more prominent as compared with the result in Fig. 2c.

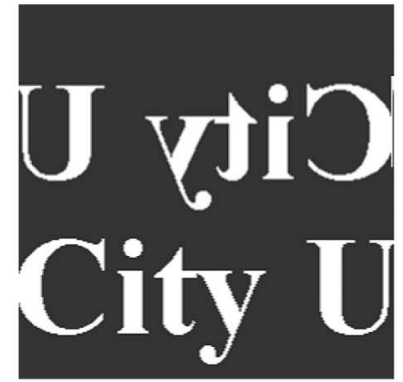

a

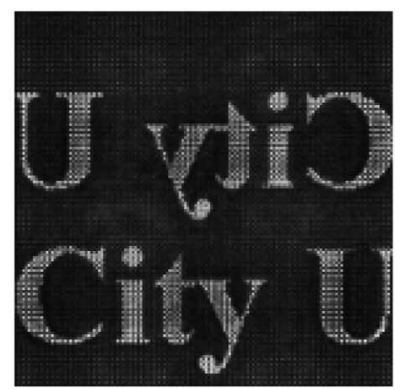

b

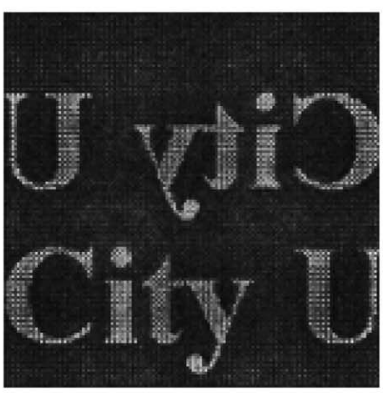

c

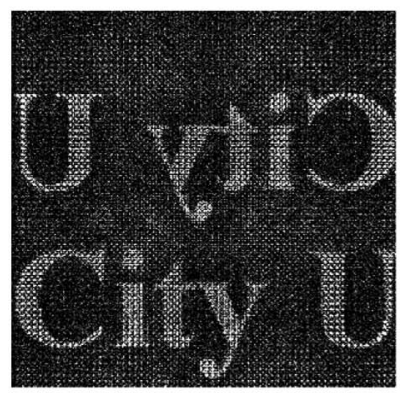

d

Fig. 2. a. Planar image positioned at $0.5 \mathrm{~m}$ from the hologram. b. Numerical reconstructed image of the binary hologram representing the image in a. The binary hologram is generated with the method in [15]. c. Numerical reconstructed image of the binary hologram representing the image in a. The binary hologram is generated with the method in [15] and contaminated with $20 \%$ of random noise. d. Numerical reconstructed image of the binary hologram representing the image in a. The binary hologram is generated with the method in [15] and contaminated with $30 \%$ of random noise.

\section{Proposed Method for Embedding Intensity Image(s) into the Binary Hologram}

The above findings have suggested that intensity image(s) can be embedded into a binary hologram. The 
Table 1. Optical Setting Adopted in the Hologram Generation Process
Hologram size

Pixel size of the hologram

Wavelength of light

Angle of illumination of $R(y)$

Downsampling factor $M$
$2048 \times 2048$ pixels

$7 \mu \mathrm{m}$ square

$650 \mathrm{~nm}$

$1.2 \mathrm{deg}$

16 embedded intensity image would be considered as noise associated with the hologram. However, there are three major problems with this straightforward approach. First, the data size of a moderate size, gray-level image is rather large. For example, to embed a $512 \times 512,8$ bit image containing 2048 Kbits of data, almost half of the area in a $2048 \times 2048$ binary hologram will be affected. Second, if the image is embedded on evenly spaced sample points in the hologram, unauthorized users can easily retrieve or tamper the hidden data. Furthermore, when a certain part of the hologram is subject to block error (i.e., damage to a connected region), the part of the embedded image within the damaged region may be degraded. To overcome these three problems, we propose the image-hiding method as shown in Fig. 3.

To begin with, the intensity image to be embedded is first binarized with error diffusion. This step reduces the representation of each pixel to 1 bit, but still preserves favorable visual quality on the intensity image. Each binarized pixel in the latter is embedded by replacing a corresponding pixel in the hologram. To address the second and the third problems, we have adopted a random-walk mechanism in the embedding process to disperse the pixels in the block error to scattered locations in the image.

To conduct the process, the binary pixels in the image are organized into an image sequence $I_{S}$ following a left to right, top to bottom order. Suppose the vertical and horizontal extents of the image are $M$ and $N$, respectively; the sequence is given by $I_{S}=\left[i_{0}, i_{1}, \ldots i_{j}, \ldots, i_{M N}\right]$, where $i_{j}$ denotes the value $(0$ or 1$)$ of the jjth binary pixel.

Next, a location sequence $P=\left[p_{0}, p_{1}, \ldots, p_{j}, \ldots p_{M N}\right]$ of identical length as the image sequence is generated with a random number generator. Each

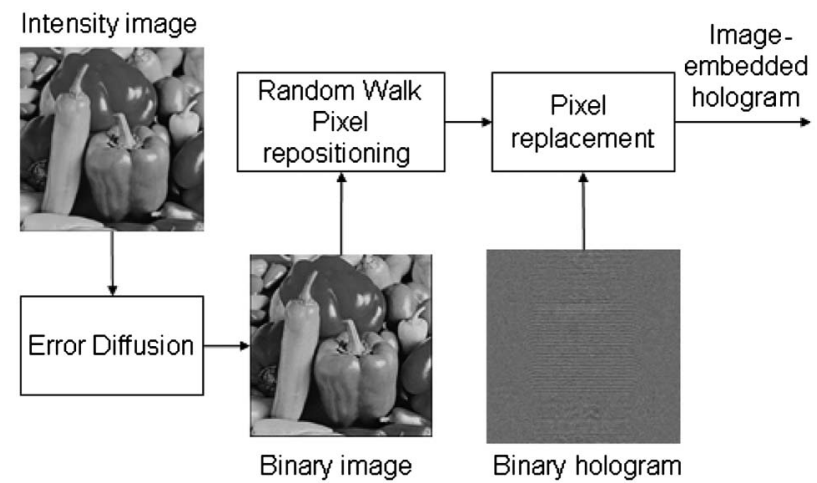

Fig. 3. Proposed intensity image-embedded binary hologram method. member in $P$ is an ordered pair $p_{j}=\left(s_{j}, t_{j}\right)$ (where $0 \leq$ $s_{j}<X$ and $0 \leq t_{j}<Y$ ) pointing to a unique position (defined by the horizontal and vertical coordinates) in the binary hologram. The sequence of random numbers is uniquely governed by a fixed number called a seed (denoted by SD) so that each value of the seed corresponds to a unique sequence $P$. In the embedding process, each hologram pixel at $p_{j}=$ $\left(s_{j}, t_{j}\right)$ is replaced by the image pixel $i_{j}$ with the forward mapping function given by

$$
H\left(s_{j}, t_{j}\right)=i_{j}
$$

As an example, we shall illustrate the random-walk embedding process with a small $2 \times 2$ image containing 4 pixels represented by the sequence $I_{S}=\left[i_{0}, i_{1}, i_{2}, i_{3}\right]$. First, a sequence of four random coordinates $P=[(2,514),(1415,162),(590,786)$, $(557,2047)]$ is generated based on a random number generator and a seed point. Next, each pixel in $I_{S}$ is taken to replace the hologram pixel at the position given by the coordinate of the corresponding member
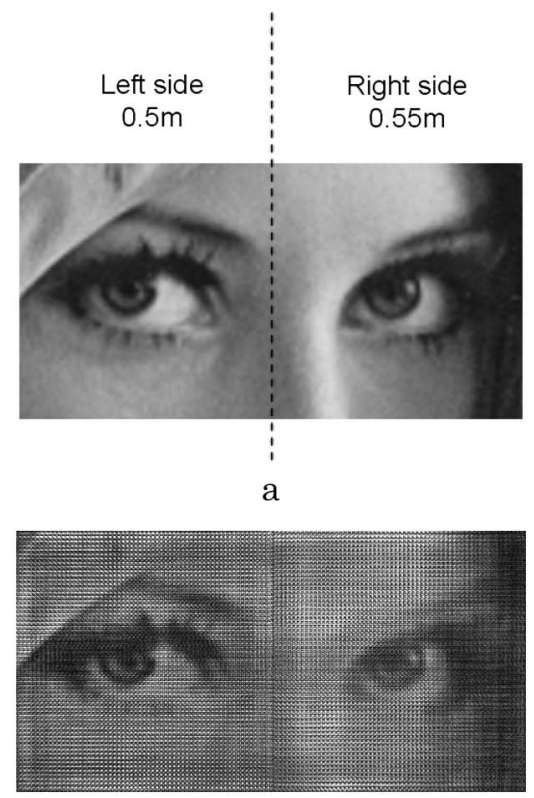

b

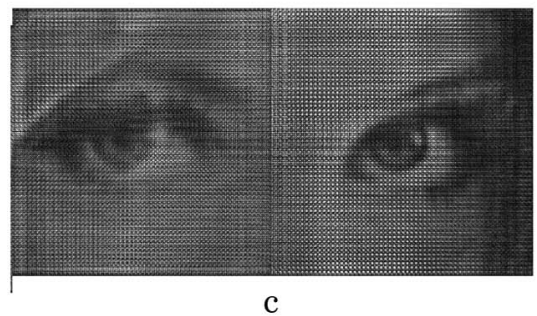

Fig. 4. a. Double depth image evenly divided into left and right sections, located at 0.5 and $0.55 \mathrm{~m}$ from the hologram, respectively. b. Numerical reconstructed image of the off-axis binary hologram at $0.5 \mathrm{~m}$ representing the double depth image in a. c. Numerical reconstructed image of the off-axis binary hologram at $0.55 \mathrm{~m}$ representing the double image in a. 
in $P$, i.e., $H(2,514)=i_{0}, \quad H(1415,162)=i_{1}$, $H(590,786)=i_{2}$, and $H(557,2047)=i_{3}$.

To retrieve the hidden image, an identical random number generator and seed value are employed to generate the sequence $P$. From the latter, the image sequence $I_{S}$ can be easily recovered by the inverse mapping function as

$$
i_{j}=H\left(s_{j}, t_{j}\right)
$$

It can be inferred from Eqs. (9) and (10) that generating the forward mapping function is equivalent to a scrambling process and converts the hidden image to a form which is difficult, if not impossible, to be retrieved by unauthorized users. Reconstruction of the hidden image required the availability of both the random number generator, together with the exact seed value, which can be taken as the descrambling key.

\section{Experimental Evaluation}

The double depth image shown in Fig. $4 \mathrm{a}$ is employed as a test sample to demonstrate the performance of our proposed method. The image is evenly divided into in a left and a right side located at 0.5 and $0.55 \mathrm{~m}$ from the hologram, respectively. The image is first downsampled by a factor of 16 according to Eqs. (2)(5) and converted to an off-axis binary hologram based on Eqs. (7) and (8) and with the optical setting in Table 1 . The numerical reconstructed images at the two depth values are shown in Figs. $4 \mathrm{~b}$ and $4 \mathrm{c}$. We observe that each side of the reconstructed image is similar to the source image when it is in focus.

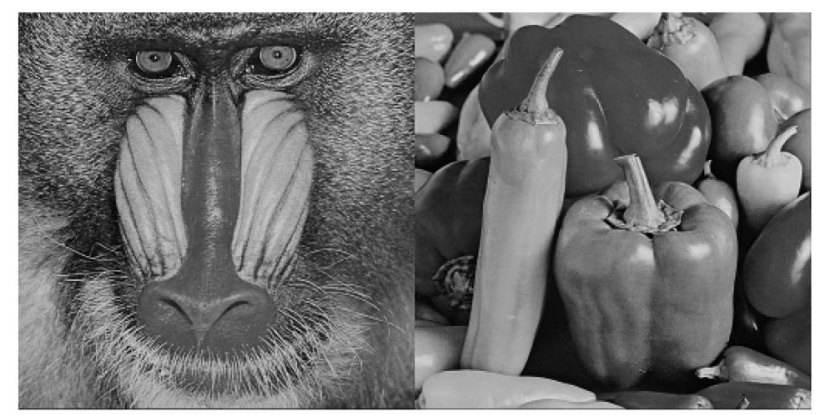

a

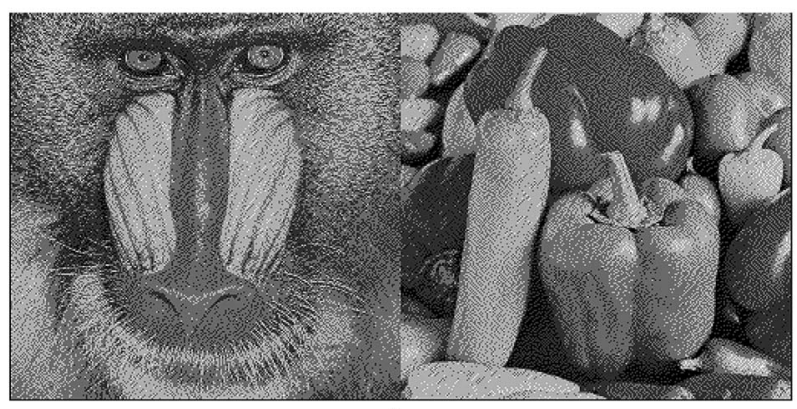

$\mathrm{b}$

Fig. 5. a. Image to be embedded into the off-axis binary hologram representing the double depth image in Fig. $\underline{4 a}$. b. Binarization of the image in Fig. 4 a with error diffusion.
Next we proceed to embed the image shown in Fig. 5a, which has a horizontal and vertical extents of 1024 and 512 pixels, respectively, into the binary hologram. Error diffusion is applied to convert the image into a binary form as shown in Fig. $5 \mathrm{~b}$ and organize it into a binary sequence $I_{S}$.

A location sequence $P$ is then generated with a random number generator based on an arbitrary seed value $\mathrm{SD}=0$. Each bit member $i_{j}$ in $I_{S}$ is taken to replace the corresponding pixel $H\left(s_{j}, t_{j}\right)$ in the binary hologram. Reconstructions of the resulting hologram, i.e., the hologram embedded with the hidden image of the two depth planes, are shown in Figs. $6 \mathrm{a}$ and $6 \mathrm{~b}$. Comparing Fig. $\underline{4 \mathrm{~b}}$ with Fig. $\underline{6 \mathrm{a}}$ and Fig. $\underline{4 \mathrm{c}}$ with Fig. $6 \mathrm{~b}$, no obvious visual changes in the reconstructed images are observed. The similarity between the focused part of the images in Figs. $4 \mathrm{~b}$ and $6 \mathrm{a}$ (the left half-planes) and between $4 \mathrm{c}$ and $\overline{6 \mathrm{~b}}$ (the right half-planes) are 29.55 and $31 . \overline{30} \mathrm{~dB}$, respectively, well above the acceptable typical PSNR of $28 \mathrm{~dB}$ mentioned earlier on visual images. Subsequent application of Eq. (10) recovers the hidden

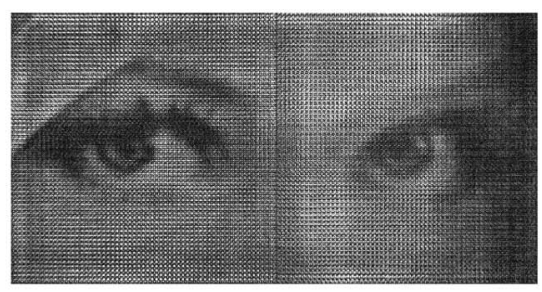

a

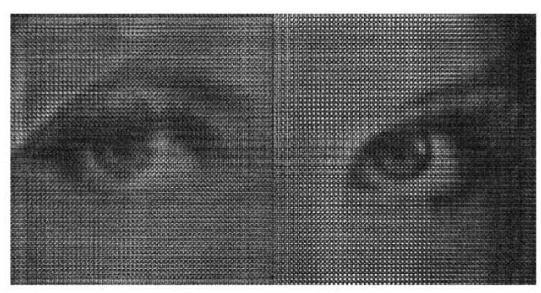

$\mathrm{b}$

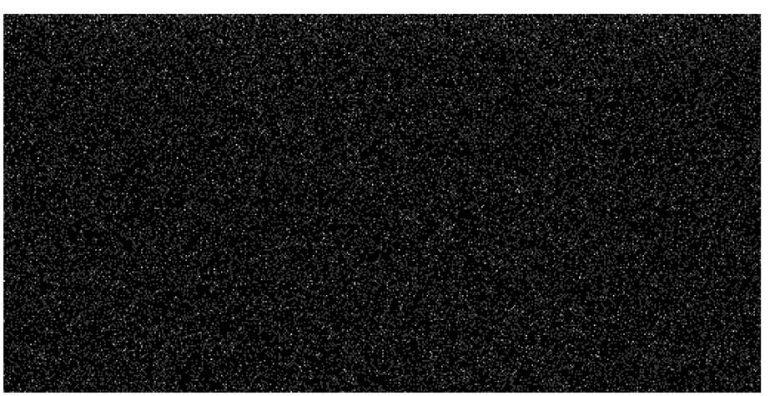

C

Fig. 6. a. Numerical reconstructed image of the off-axis binary hologram at $0.5 \mathrm{~m}$ representing the double depth image in Fig. $4 \mathrm{a}$. The image in Fig. 5b is embedded into the binary hologram based on the proposed method. b. Numerical reconstructed image of the off-axis binary hologram at $0.55 \mathrm{~m}$ representing the double image in Fig. 4a. The image in Fig. 5b is embedded into the binary hologram based on the proposed method. c. Extracted image from the binary hologram (that has been embedded with the image in Fig. $5 \mathrm{~b}$ with our proposed method and a seed value $\mathrm{SD}=0$ ), based on a different seed value $\mathrm{SD}=1$. 
image completely (identical to the source image shown in Fig. 5b). With a commodity PC of moderate computing power, the image embedding and retrieving processes each take less than $1.5 \mathrm{~ms}$ to accomplish. Next we study the effect of the seed point by retrieving the embedded image from the binary hologram with a different seed value, $\mathrm{SD}=1$. The retrieved image is shown in Fig. $6 \mathrm{c}$, showing that the content is a random noise image that is totally different from the embedded image in Fig. 5b. Seed values other than the one used to embed the image have been studied, and they all result in random noise images that are similar to the one in Fig. $\underline{6 c}$.

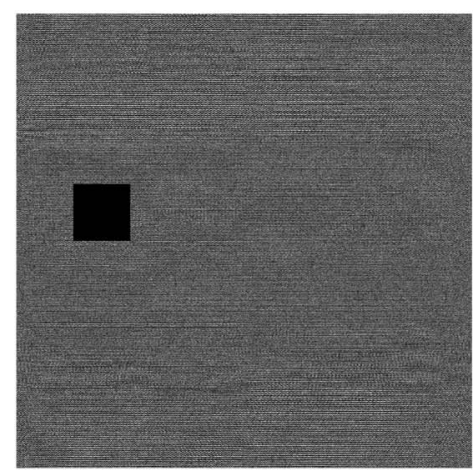

a
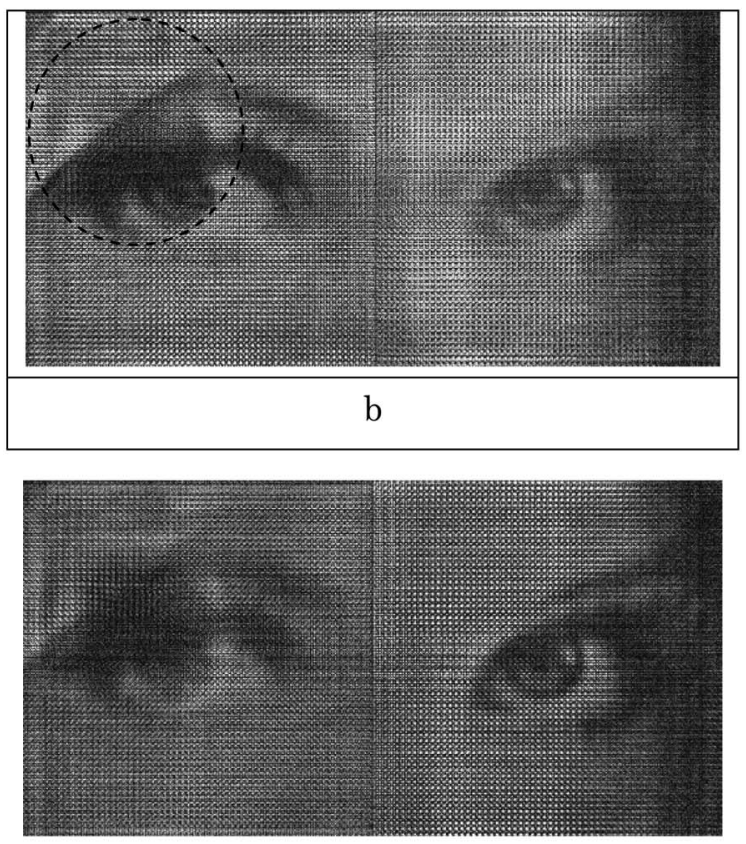

C

Fig. 7. a. Blanking a rectangular region in the off-axis binary hologram representing the double depth image in Fig. 4a, and which has been embedded with the binary image in Fig. $5 \mathrm{~b}$. b. Numerical reconstructed image of the damaged and noise-contaminated offaxis binary hologram in a., at $0.5 \mathrm{~m}$ representing the double image in Fig. 4a. The image in Fig. 5a is embedded into the binary hologram based on the proposed method. c. Numerical reconstructed image of the damaged and noise-contaminated off-axis binary hologram in a., at $0.55 \mathrm{~m}$ representing the double image in Fig. $\underline{4 a}$. The image in Fig. 5a is embedded into the binary hologram based on the proposed method.

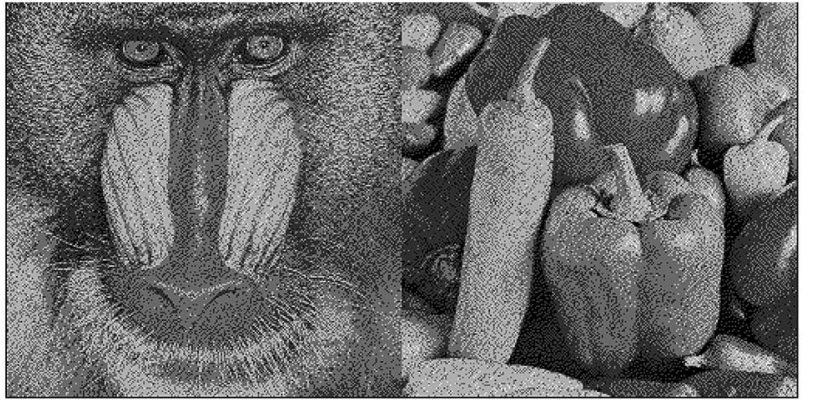

Fig. 8. Extracted embedded image from the damaged and noisecontaminated binary hologram in Fig. 7a.

To demonstrate the robustness of our proposed method in preserving the pictorial content recorded in the binary hologram when the latter is damaged, we blank out a $256 \times 256$ rectangular region (containing 65,536 hologram pixels, around $1.56 \%$ of the total hologram pixels) on the image-embedded binary hologram in the previous example. A total of 32,337 , constituting to about $0.77 \%$ of the total pixels in the hologram, has been complemented (changed from white to black) with the blanking operation. In addition, about $2 \%$ of noise (i.e., about 84,000 binary pixels are complemented) are added to the hologram. The damaged hologram is shown in Fig. 7a. The numerical reconstructed images at the two depth planes are shown in Figs. 7b and 7c. We observe that, apart from a minor degradation around the region marked by the dotted circle in Fig. 7b, the reconstructed images are similar to the one corresponding to the undamaged hologram. The embedded image extracted from the damaged and noise-contaminated hologram is shown in Fig. 8. We also find that the total number of pixels that have been changed from black to white, or vice versa, is 14,120 , which is about $2.69 \%$ of the size of the embedded image. We note that the number of pixels that have been changed is much less than the number of damaged (or noisecontaminated) pixels in the hologram. This is due to the random-walk process, which disperses the embedded pixels to different locations in the entire hologram rather than concentrating in a specific area or sampling lattice. Visually, we observe that the blanking of the rectangular region and the noise contamination only leads to scattering noise points, otherwise undetectable visually on the retrieved image.

\section{Conclusion}

A binary Fresnel hologram can be generated to preserve favorable visual quality on the object scene by first downsampling the latter image with a grid-cross lattice and converting the hologram to a bilevel representation via sign binarization. In this paper, we have demonstrated that a binary hologram generated with such means is highly resistant to noise contamination. On this basis, we have proposed a method to embed a hidden intensity image into the binary hologram. In essence, the hidden image 
is first binarized into a one bit representation with error diffusion. Next each pixel in the binarized image is taken to replace a corresponding pixel in the hologram based on a location mapping sequence derived from a random number generator and a seed value. Recovery of the hidden image requires an identical random number generator together with the correct seed value. Experimental results have demonstrated that the hidden image can be recovered to a good extent even if the binary hologram is damaged and contaminated with noise. Based on the findings in this paper, further research can be conducted to extend, with suitable modifications, our proposed image embedding scheme on binary holograms that are generated with different means, for example the one reported in [23].

This work is supported by the Chinese Academy of Sciences Visiting Professorships for Senior International Scientists Program under grant 2010T2G17.

\section{References}

1. T.-C. Poon, ed., Digital Holography and Three-Dimensional Display: Principles and Applications (Springer, 2006).

2. L. C. Ferri, "Visualization of 3D information with digital holography using laser printers," Comput. Graph. 25, 309-321 (2001).

3. H. Yoshikawa and M. Tachinami, "Development of direct fringe printer for computer-generated holograms," Proc. SPIE 5742, 259-266 (2005)

4. A. W. Lohmann and D. P. Paris, "Binary Fraunhofer holograms, generated by computer," Appl. Opt. 6, 1739-1748 (1967).

5. B. R. Brown and A. W. Lohmann, "Computer-generated binary holograms,” IBM J. Res. Devel. 13, 160-168 (1969).

6. M. P. Chang and O. K. Ersoy, "Iterative interlacing error diffusion for synthesis of computer-generated holograms," Appl. Opt. 32, 3122-3129 (1993).

7. E. Zhang, S. Noehte, C. H. Dietrich, and R. Männer, "Gradual and random binarization of gray-scale holograms," Appl. Opt. 34, 5987-5995 (1995).

8. B. B. Chhetri, S. Yang, and T. Shimomura, "Iterative stepwise binarization of digital amplitude holograms with added energy to the signal window," Opt. Eng. 40, 2718-2725 (2001).
9. R. W. Floyd and L. Steinberg, "An adaptive algorithm for spatial grey scale," Proc. Soc. Inf. Disp 17, 75-77 (1976).

10. R. Eschbach, "Comparison of error diffusion methods for computer-generated holograms," Appl. Opt. 30, 3702-3710 (1991).

11. R. Eschbach and Z. Fan, "Complex-valued error diffusion for off-axis computer-generated holograms," Appl. Opt. 32, 3130-3136 (1993).

12. R. L. Easton, R. Eschbach, and R. Nagarajan, "Error diffusion in cell-oriented Fourier-transform computer-generated holograms to compensate for printing constraints," J. Mod. Opt. 43, 1219-1236 (1996).

13. F. Fetthauer, S. Weissbach, and O. Bryngdahl, "Computergenerated Fresnel holograms: quantization with the error diffusion algorithm," Opt. Commun. 114, 230-234 (1995).

14. P. Tsang, T.-C. Poon, W.-K. Cheung, and J.-P. Liu, "Computer generation of binary Fresnel holography," Appl. Opt. 50, B88-B95 (2011).

15. W. K. Cheung, P. Tsang, T.-C. Poon, and C.-H. Zhou, "Enhanced method for the generation of binary Fresnel holograms based on grid-cross downsampling," Chinese Opt. Lett. 9, 120005 (2011)

16. C. Martinez, O. Lemonnier, F. Laulagnet, A. Fargeix, F. Tissot, and M. Armand, "Complementary computer generated holography for aesthetic watermarking," Opt. Express 20, 5547-5556 (2012).

17. K. Tanaka, "Embedding of computer-generated hologram in a dithered image," Appl. Opt. 50, H315-H326 (2011).

18. X. Zhou, L. Chen, and J. Shao, "Investigation of digital hologram watermarking with double binary phase encoding," Proc. SPIE 5636, 220-228 (2005).

19. A. Yoshinao, "Watermarking technique using computer generated holograms," Electron. Commun. Jpn. III 84, 21-31 (2001).

20. X. Shi and D. Zhao, "Image hiding in Fourier domain by use of joint transform correlator architecture and holographic technique," Appl. Opt. 50, 766-772 (2011).

21. N. K. Nishchal, T. Pitkaaho, and T. J. Naughton, "Digital Fresnel hologram watermarking," in Proceedings of the 9th Euro-American Workshop on Information Optics (WIO) (IEEE, 2010), pp. 1-3.

22. S. Deng, L. Liu, H. Lang, W. Pan, and D. Zhao, "Hiding an image in cascaded Fresnel digital holograms," Chin. Opt. Lett. 4, 268-271 (2006).

23. P. Tsang, W.-K. Cheung, T.-C. Poon, and J.-P. Liu, "An enhanced method for generation of binary Fresnel hologram based on adaptive and uniform grid-cross down-sampling," Opt. Commun. 285, 4027-4032 (2012). 